\title{
Trends in Hospitalization for Opioid Overdose among Rural Compared to Urban Residents of the United States, 2007-2014
}

\author{
Hilary Mosher, MFA, MD ${ }^{1,2,3 *}$, Yunshou Zhou, PhD³, Andrew L. Thurman, PhD ${ }^{1,2}$, \\ Mary Vaughan Sarrazin, PhD $^{1,2,3}$, Michael E. Ohl, MD, MSPH ${ }^{1,2,3}$
}

\begin{abstract}
'Veterans Affairs Office of Rural Health, Veterans Rural Health Resource Center-Central Region, lowa City Veterans Affairs Healthcare System, lowa City, lowa; ${ }^{2}$ The Comprehensive Access and Delivery Research and Evaluation Center at the lowa City Veterans Affairs Healthcare System, lowa City, lowa; ${ }^{3}$ The Department of Internal Medicine, Carver College of Medicine, University of lowa, lowa City, lowa.
\end{abstract}

Hospitalizations and deaths due to opioid overdose have increased over the last decades. We used data from the National Inpatient Sample and the American Community Survey to describe trends in hospitalization rates for opioid overdose among rural residents compared with urban residents in the United States from 2007 to 2014. Hospitalization rates for heroin overdose increased in all years and were higher in urban residents compared with rural residents (5.5 per 100,000 in large urban populations vs 2.1 per 100,000 in rural populations in 2014). In contrast, hospital- ization rates for prescription opioid overdose were $20 \%$ to $30 \%$ higher in rural populations compared with large urban populations between 2007 and 2012, before declining in rural populations in 2013 and 2014. The proportion of rural patients admitted for overdose who are cared for in urban hospitals increased from $23.1 \%$ in 2007 to $41.2 \%$ in 2014. These trends are clinically relevant as rural patients and urban patients may have different discharge needs. Journal of Hospital Medicine 2017;12:925-929. Published online first August 23, 2017. (C) 2017 Society of Hospital Medicine

\section{BACKGROUND}

Hospitalizations and deaths due to opioid overdose have increased over the last decades, straining the healthcare system and generating substantial costs. ${ }^{1-4}$ Hospitalizations for overdose also represent opportunities to intervene in the opioid epidemic by linking patients to resources for nonpharmacologic chronic pain treatment resources or substance use treatment services during and following hospitalization. ${ }^{5,6}$ Studies of trends in the frequency of hospitalizations for opioid overdose in rural and urban areas are necessary to inform planning and resource allocation for inpatient and postdischarge transitional care.

Nonmedical opioid use and opioid-related deaths and injuries appear to be higher in rural areas. ${ }^{7,8}$ As well, rural areas tend to be more under-resourced in terms of substance abuse treatment and chronic pain specialty services. ${ }^{9,10}$ Contemporaneous with rising opioid use has been an increasing trend of rural hospital closures. ${ }^{11}$ This may compound the impact of opioid-related hospitalizations on remaining rural hospitals and lead to increasing reliance on more distant, urban hospitals to treat and discharge patients with overdoses. Rural residents who are admitted or transferred to urban hospitals may face distinct challenges. Similarly, urban hospitals may struggle during discharge planning to link patients to substance use

\footnotetext{
*Address for correspondence and reprint requests: Hilary Mosher, MFA, MD, Iowa City VA Healthcare System, 601 Highway 6 West, Mailstop 152, lowa City, IA 52246-2208; Telephone: 319-338-0581, extension 7723; Fax: 319-8874932; E-mail: hilary.mosher@va.gov

Additional Supporting Information may be found in the online version of this article.

Received: January 10, 2017; Revised: April 29, 2017;

Accepted: May 17, 2017
}

Society of Hospital Medicine DOI: 10.12788/jhm.2793 treatment services in less familiar rural communities.

To better define the differential impact of the opioid epidemic based on patient rurality, we described trends in rates of hospitalization for opioid overdose among rural residents compared with urban residents of the United States. We separated hospitalizations into those due to overdose of prescription opioids, and those related to heroin. Among rural residents who overdosed on opioids, we examined trends in admission to rural versus urban hospitals.

\section{METHODS}

\section{Data Source}

We analyzed data from the National Inpatient Sample (NIS) from 2007 to 2014, developed by the Healthcare Cost and Utilization Project (HCUP). NIS yields nationally representative estimates of inpatient stays in community hospitals in the United States, regardless of payer. Rehabilitation and long-term care hospital stays are excluded. Prior to 2012, NIS included data on all discharges from a $20 \%$ sample of hospitals. Beginning in 2012, NIS included a 20\% sample of discharges from all HCUP hospitals. We used weights to estimate trends in the total number of hospital admissions for heroin and prescription opioid overdose (POD) in the US by year, accounting for the change in sampling design in 2012 as recommended by HCUP. Standard errors for estimates accounted for the complex sample design. ${ }^{12}$ We used data from the US Census American Community Survey on the US population in rural versus urban areas for each year to calculate overdose admission rates per 100,000 residents.

\section{Target Population}

Following methods applied in previous analyses of NIS data, ${ }^{1,4,13}$ we identified hospitalizations for heroin or POD 

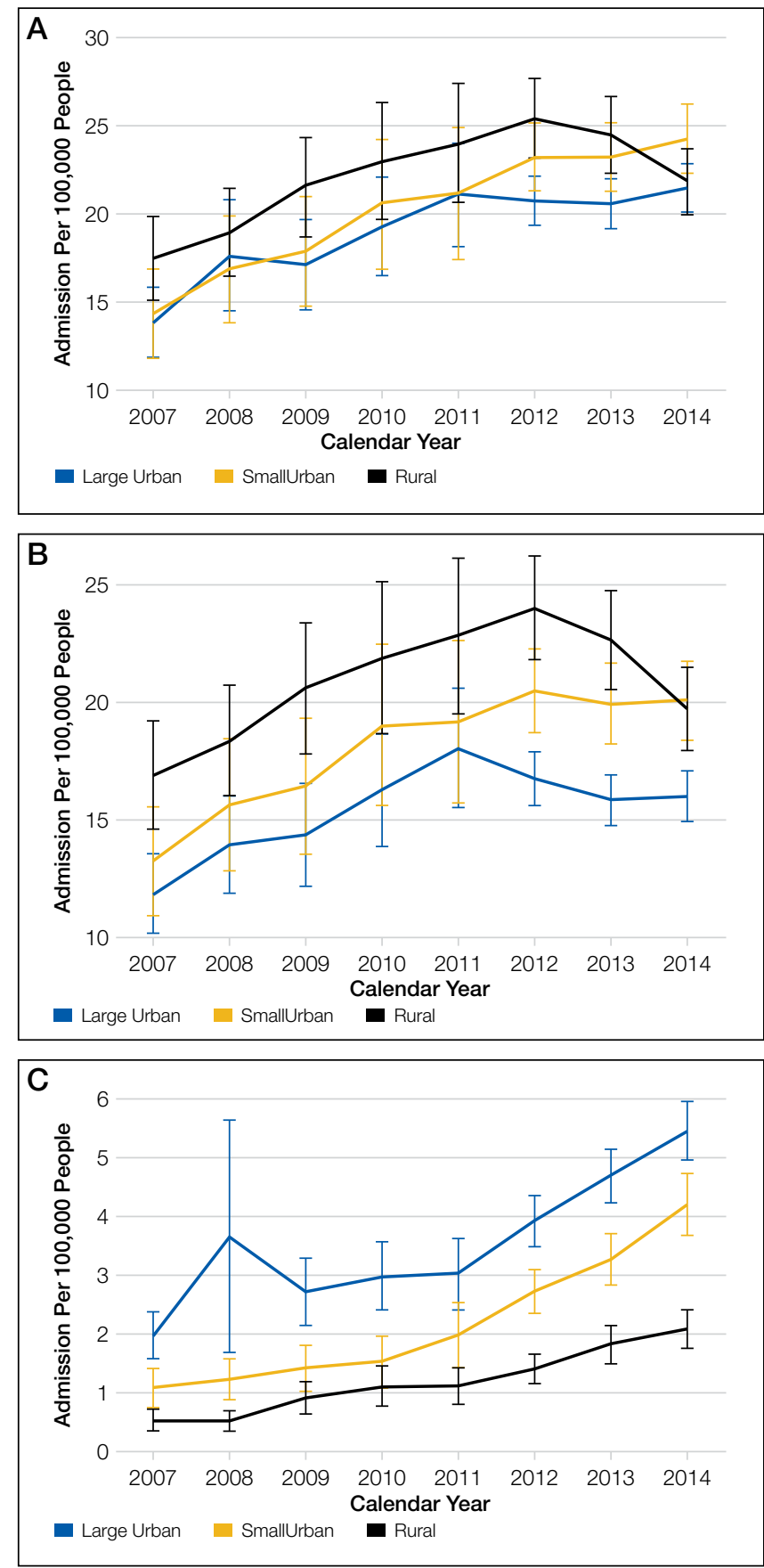

FIG 1. (A) Total opiod overdose admission trends by rurality, 2007-2014. (B) POD admission trends by rurality, 2007-2014. (C) HOD admission trends by rurality, 2007-2014.

NOTE: Abbreviations: HOD, heroin overdose; POD, prescription opioid overdose.

based on International Classification of Diseases 9th Clinical Modification (ICD-9-CM) codes. We use the lay term "overdose" to refer to admissions defined by the medical term "poisoning." In each year between 2007 and 2013, we determined the total number of admissions due to heroin or prescription opioid by considering ICD-9CM codes 965.00 (poisoning by opium), 965.01 (poisoning by heroin), or 965.09 (poisoning by other opiates and related narcotics); or E code E850.0 (accidental poisoning by heroin); or 850.2 (accidental poisoning by opiates and related narcotics) in any position. We defined admissions for heroin overdose (HOD) as 965.01 or E code of E850.0 in any position, and admissions for POD not related to heroin as 965.00 , or 965.09 , or E code 850.2 in any position excluding admissions with any heroin-related code 965.01 or E code E850.0 or E935.0 (adverse effects of heroin). We excluded hospitalizations in which a patient was transferred out to another acute care facility to avoid duplicate counting.

\section{Analysis}

We classified these admissions based on patient residence in a rural versus urban area. NIS contained a variable representing rural versus urban patient residence based on the county-level framework maintained by the Office of Management and Budget, supplemented with information from Urban Influence Codes developed by the Economic Research Service of the US Department of Agriculture. ${ }^{14} \mathrm{We}$ used this information to create a 3-level variable for patient residence: rural (ie, nonmetropolitan areas with a population less than 50,000), small metropolitan (ie, metropolitan areas with a population of 50,000-999,999), and large metropolitan (ie, metropolitan areas with a population of 1,000,000 or greater). We explored further separating categories (eg, breaking rural into micropolitan population centers and other), but this did not further discriminate admission rates.

For each study year, we combined results on overdose admissions with data on the total populations for each of these 3 areas in the US based on American Community Survey data in order to calculate rates of each type of admission per 100,000 persons. To compare pharmaceutical opioids to heroin, we examined pharmaceutical-only overdoses and heroin-only overdoses. We also examined patient age, sex, race and/or ethnicity, and whether they were admitted to a rural or urban hospital based on the hospital location code contained in NIS, and compared these characteristics across residence categories; we presented characteristics for years 2012 to 2014 combined as recent characteristics are most relevant.

The authors had full access to and take full responsibility for the integrity of the data. All analyses were conducted using SAS statistical software version 9.2 (SAS Institute, Cary, North Carolina). The study was reviewed by the University of Iowa Institutional Review Board and the Iowa City Veterans Affairs Healthcare System Research and Development Committee and was judged human subject research exempt.

\section{RESULTS}

\section{Characteristics of Patients with Opioids Overdose Admissions}

An estimated 43,935 individuals experienced an opioid overdose-related hospitalization in the US in 2007 and 71,280 in 2014. Characteristics of admitted patients varied by residence: a greater proportion of rural patients in older age categories were female $(57.3 \%)$ and were Caucasian $(90.1 \%$; Table). The overwhelming majority of large and small urban 
TABLE. Characteristics of Patients Hospitalized for Opioid- or Heroin-Related Causes, 2012-2014

\begin{tabular}{|c|c|c|c|}
\hline \multirow[b]{2}{*}{ Characteristics } & \multicolumn{3}{|c|}{ Patient Residence } \\
\hline & Large Urban & Small Urban & Rural \\
\hline & $\mathrm{N}(\%)$ & $\mathrm{N}(\%)$ & $\mathrm{N}(\%)$ \\
\hline \multicolumn{4}{|l|}{ Age, years } \\
\hline$\leq 25$ & $14,100(13.13 \%)$ & $8345(12.59 \%)$ & $3835(10.48 \%)$ \\
\hline $26-45$ & 33,995 (31.66\%) & 20,475 (30.89\%) & $11,480(31.36 \%)$ \\
\hline $46-65$ & $44,245(41.21 \%)$ & $27,585(41.62 \%)$ & $15,565(42.52 \%)$ \\
\hline$>65$ & $15,025(13.99 \%)$ & $9875(14.90 \%)$ & 5725 (15.64\%) \\
\hline Female & $55,215(51.36 \%)$ & $7368(55.52 \%)$ & $20,995(57.31 \%)$ \\
\hline \multicolumn{4}{|l|}{ Race } \\
\hline Caucasian & $76,560(74.65 \%)$ & $52,210(83.70 \%)$ & $30,455(90.13 \%)$ \\
\hline African American & $13,350(13.02 \%)$ & $4165(6.68 \%)$ & $1055(2.12 \%)$ \\
\hline Hispanic & $8505(8.29 \%)$ & $4180(6.70 \%)$ & $1000(2.96 \%)$ \\
\hline Other & $4140(4.04 \%)$ & $1825(2.92 \%)$ & $1280(3.79 \%)$ \\
\hline Admitted to an urban hospital & $106,855(99.35 \%)$ & $65,230(98.28 \%)$ & $13,645(37.24 \%)$ \\
\hline \multicolumn{4}{|l|}{ Type of admission } \\
\hline POD & $83,370(77.53 \%)$ & $56,800(85.59 \%)$ & $33,930(92.60 \%)$ \\
\hline HOD & $24,165(22.47 \%)$ & $9560(14.41 \%)$ & $2710(7.40 \%)$ \\
\hline Admitted as transfer from another hospital & $3755(3.52 \%)$ & $2670(4.04 \%)$ & 5225 (14.34\%) \\
\hline Died during hospitalization & 3605 (3.36\%) & 2045 (3.08\%) & $850(2.32 \%)$ \\
\hline
\end{tabular}

residence patients were admitted to urban hospitals $(99.4 \%$ and $98.3 \%$, respectively) compared with $37.2 \%$ of rural patients. The proportion of total opioid overdose admissions due to prescription opioids was higher among rural than urban residents $(92.6 \%$ for rural residents, $85.6 \%$ small urban, and $77.5 \%$ large urban). The proportion of large urban $(3.5 \%)$ and small urban $(4.0 \%)$ patients admitted as hospital transfers was small in comparison to $14.3 \%$ of rural patients. The proportion of admitted patients who died in the hospital varied by patient residence (Table).

Opioid Overdose Admission Trends by Patient Residence Opioid admission rates increased between 2007 and 2011 in all groups; trends then diverged (panel $A$ in Figure 1). In 2007, 13.8 (95\% confidence interval [CI], 11.9-15.8) people per 100,000 had opioid overdose admissions among large urban residents, compared with 17.5 (95\% CI, 15.1-19.8) among rural residents. By 2014, these rates were $21.5(95 \%$ CI, 20.1-22.9) among urban residents, and 21.8 (95\% CI, 19.9-23.7) among rural residents. Rates for POD admissions followed a similar pattern. POD admission rates rose in all groups until 2011 and then started to decline among large urban residents while continuing to increase among small urban residents. Among rural residents, POD admission rates peaked in 2012 and then declined in 2013 and again in 2014 (panel B in Figure 1). Rates for HOD admissions were highest among urban residents during each study year, increasing from 2.0 per 100,000 residents (95\% CI, 1.6-2.4) in 2007 to 5.5 (95\% CI, 5.0-6.0) in 2014. Among rural residents, the rate increased from 0.5 (95\% CI, 0.3-0.7) to 2.1 (95\% CI, 1.8-2.4) over the same time period (panel C in Figure 1).

\section{Opioid Overdose Admissions among Rural Residents to Urban and Rural Hospitals}

The estimated total number of patients residing in rural areas who were admitted with opioid overdose to rural hospitals decreased from 6731 in 2007 to 6550 in 2014. Rural patients admitted to urban hospitals increased from 2014 to 4595 over that same time period; the proportion of rural patients admitted to urban hospitals increased from $23.1 \%$ in 2007 to $41.2 \%$ in 2014 (Figure 2).

\section{DISCUSSION}

Up until 2013, hospital admissions for POD occurred at a higher rate among rural US residents than their urban counterparts. Rates of admission of rural residents for POD have decreased since 2012; a similar trend was not observed among urban residents. Over this same interval, rates of hospitalization for HOD among rural residents continued to increase.

Hospital admission is one sequela of harm related to opioid use: patients experiencing opioid overdose or poisoning may be treated by emergency responders, in emergency 


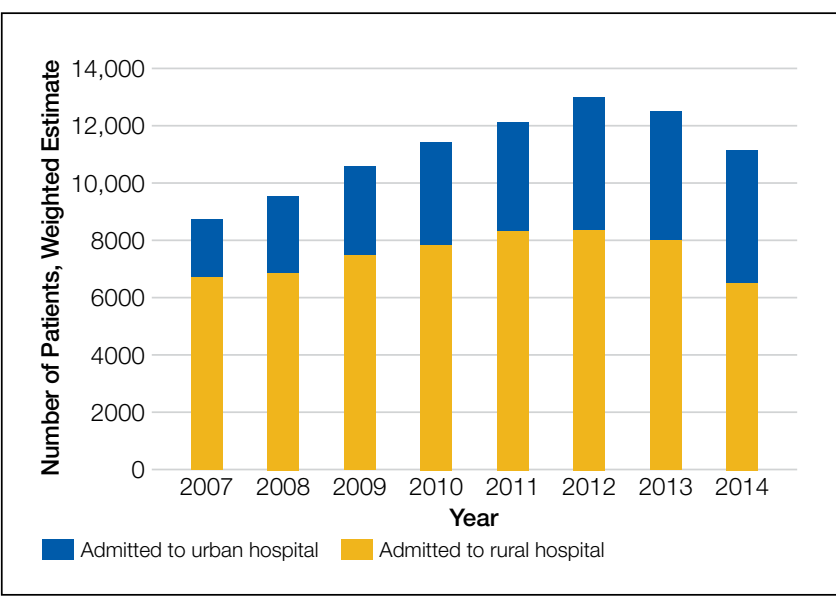

FIG 2. An increasing proportion of rural patients were admitted to urban hospitals, 2007-2014.

departments or on observation status, or may die prior to receiving medical attention or presenting for hospital admission. Factors potentially driving the trends described include patient behaviors, opioid availability, prehospital and hospital treatment practices, and hospital closures. Recent work describing increased opioid overdose deaths ${ }^{15}$ and high opioid-related mortality in rural areas ${ }^{16}$ suggests that overdose admission and death rates may be divergent. Changing policies governing naloxone availability and administration ${ }^{17}$ and ongoing trends in rural hospital closures ${ }^{11}$ may differentially affect the rates at which rural and urban residents who experience overdose are hospitalized.

Hospital admission also represents a potential point-ofentry into subsequent treatment to reduce risk of further opioid-related harms. Decreasing rates of admission could conceivably result in decreasing opportunities to engage in care. Rural and urban patient populations are distinct; an understanding of these distinctions may help to inform how hospitals structure inpatient treatment and discharge planning for overdose patients. Overdose is likely to suggest either an underlying substance use disorder or a chronic pain condition requiring risky levels of prescribed opioids, and therefore is indicative of a persistent condition requiring follow-up care. Thus, there is a need for treatment models and transition care systems aimed at providing adequate care for these populations both in the acute setting and following hospital discharge. The increasing proportion of rural residents admitted to urban hospitals with opioid overdoses highlights the need for urban hospitals to develop relationships with substance use treatment and chronic pain services in rural areas to facilitate linkage to treatment at discharge.

Limitations of this study include the use of ICD-9-CM codes from administrative data to identify hospitalizations for prescription opioid and heroin overdose. While we have used the common term "overdose," opioid adverse events may occasion hospitalization in the absence of overdose or as a result of patients taking opioid doses in the quantity prescribed. As such, the term overdose does not necessarily imply the behavior of intentional or unintentional excess use. Additionally, coding depends on providers diagnosing and documenting conditions and may be subject to secular trends independent of overdose prevalence. We included data through 2014, the most recent year of data available at time of analyses.

\section{CONCLUSION}

Hospitals can expect to continue to treat patients presenting with opioid overdose. As overdose is likely to suggest either an underlying substance use disorder or a chronic pain condition requiring risky levels of prescribed opioids, there will be a need for treatment models and transition care systems to provide adequate care for these populations both in the acute setting and following hospital discharge. Rates of admission among rural residents declined during the last 2 years of the study period, and rural residents who were hospitalized for opioid overdose were increasingly receiving care in urban hospitals. While factors driving these trends remain to be elucidated, the trends themselves highlight a need to consider the differential challenges facing rural and urban residents who overdose. Access to resources and transportation and other challenges are distinct in urban and rural areas, with rural areas being less likely to have providers in addiction medicine, psychiatry, and pain specialties. Efforts to address these challenges will need to explore models and solutions applicable to differentially resourced hospital and postdischarge settings.

Disclosure: The work reported here was supported by the Department of Veterans Affairs, Veterans Health Administration, Office of Rural Health, Veterans Rural Health Resource Center-Central Region, and the Health Services Research and Development Service through the Comprehensive Access and Delivery Research and Evaluation Center (HFP 04-149). This manuscript is not under review elsewhere and there is no prior publication or presentation of manuscript contents. The views expressed in this article are those of the authors and do not necessarily represent the views of the Department of Veterans Affairs. The authors report no conflict of interest in regards to this study. Data: Available to researchers with VA accreditation. Statistical Code: Available to interested readers by contacting Dr. Ohl. Protocol: Available to interested readers by contacting Dr. Ohl.

\section{References}

1. Ronan MV, Herzig SJ. Hospitalizations Related To Opioid Abuse/Dependence And Associated Serious Infections Increased Sharply, 2002-12. Health Aff (Millwood). 2016;35:832-837.

2. Florence CS, Zhou C, Luo F, Xu L. The Economic Burden of Prescription Opioid Overdose, Abuse, and Dependence in the United States, 2013. Med Care. 2016;54:901-906.

3. Jennifer PS, Michael JW, Douglas H, John M, Michael DH. The Critical Care Crisis of Opioid Overdoses in the U.S. In: C95 OUTSTANDING EPIDEMIOLOGY AND HEALTH SERVICES RESEARCH IN CRITICAL CARE: American Thoracic Society 2016 International Conference; 2016 May 13-18; San Francisco, CA:A6146-A.

4. Owens PL, Barrett ML, Weiss AJ, Washington RE, Kronick R. Hospital Inpatient Utilization Related to Opioid Overuse Among Adults, 1993-2012: Statistical Brief \#177. Healthcare Cost and Utilization Project (HCUP) Statistical Briefs; 2006. https://www.hcup-us.ahrq.gov/reports/statbriefs/sb177-Hospitalizations-for-Opioid-Overuse.jsp. Accessed January 4, 2017

5. Fanucchi L, Lofwall MR. Putting Parity into Practice - Integrating Opioid-Use Disorder Treatment into the Hospital Setting. N Engl J Med. 2016;375:811-813.

6. Liebschutz JM, Crooks D, Herman D, et al. Buprenorphine treatment for hospi- 
talized, opioid-dependent patients: a randomized clinical trial. JAMA Intern Med 2014;174:1369-1376.

7. Keyes KM, Cerda M, Brady JE, Havens JR, Galea S. Understanding the rural-urban differences in nonmedical prescription opioid use and abuse in the United States. Am J Public Health. 2014;104:e52-e59.

8. Rigg KK, Monnat SM. Urban vs. rural differences in prescription opioid misuse among adults in the United States: informing region specific drug policies and interventions. Int J Drug Policy. 2015;26:484-491.

9. Ellis AR, Konrad TR, Thomas KC, Morrissey JP. County-level estimates of mental health professional supply in the United States. Psychiatr Serv. 2009;60: 1315-1322.

10. Rosenblatt RA, Andrilla CH, Catlin M, Larson EH. Geographic and specialty distribution of US physicians trained to treat opioid use disorder. Ann Fam Med. 2015;13:23-26.

11. Kaufman BG, Thomas SR, Randolph RK, et al. The Rising Rate of Rural Hospital Closures. J Rural Health. 2016;32:35-43.
12. Houchens RL DR, A Elixhauser. Using the HCUP National Inpatient Sample to Estimate Trends: U.S. Agency for Healthcare Research and Quality; 2015. Report No.: 2006-05

13. Unick GJ, Rosenblum D, Mars S, Ciccarone D. Intertwined epidemics: national demographic trends in hospitalizations for heroin- and opioid-related overdoses, 1993-2009. PLoS One. 2013;8:e54496.

14. Urban Influence Codes. USDA, 2016. https://www.ers.usda.gov/data-products/ urban-influence-codes.aspx. Accessed January 4, 2017

15. Rudd RA, Seth P, David F, Scholl L. Increases in Drug and Opioid-Involved Overdose Deaths - United States, 2010-2015. MMWR Morb Mortal Wkly Rep. 2016;65:1445-1452.

16. Case A, Deaton A. Rising morbidity and mortality in midlife among white non-Hispanic Americans in the 21st century. Proc Natl Acad Sci U S A. 2015;112:15078-15083.

17. Davis CS, Southwell JK, Niehaus VR, Walley AY, Dailey MW. Emergency medical services naloxone access: a national systematic legal review. Acad Emerg Med. 2014;21:1173-1177. 